\title{
ON A CONJECTURE OF NITSCHE
}

\author{
GREGORY D. CROW
}

(Communicated by Peter Li)

\begin{abstract}
We show that under the hypothesis of bounded Gaussian curvature, certain minimal surfaces are in fact of finite total curvature. We can then answer the following version of a conjecture of Nitsche (J. Math. Mech. 11 (1962), 295) under the hypothesis of bounded Gaussian curvature:

Conjecture. Let $M^{2} \subset \mathbf{R}^{3}$ be a complete minimal surface such that for some height function $H$, the level sets are (compact) Jordan curves. Then $M$ is a catenoid.
\end{abstract}

Much is known about minimal surfaces that are of finite total curvature [JM, $\mathrm{O}, \mathrm{S}]$. We show that under the hypothesis of bounded Gaussian curvature, certain minimal surfaces are in fact of finite total curvature. With this information we can then answer the following version of a conjecture of Nitsche [Ni], under the hypothesis of bounded Gaussian curvature:

Conjecture. Let $M^{2} \subset \mathbf{R}^{3}$ be a complete minimal surface such that for some height function $H$, the level sets are (compact) Jordan curves. Then $M$ is a catenoid.

In [CHM, p. 1], an example is given, due to Riemann, whose level sets are only round circles and straight lines. This example shows that the conjecture would be false without the compactness hypothesis. Let $S^{2}$ denote the Riemann sphere. The conjecture (with $|K|<C$ ) follows from the following deeper therom

Theorem 1. Let $M^{2} \subset \mathbf{R}^{3}$ be a complete minimal immersion satisfying

(i) $|K|<C$ ( $M$ is of bounded Gaussian curvature).

(ii) The immersion is given by $I=\left(I_{1}, I_{2}, I_{3}\right): S^{2}-\{p, q\} \rightarrow \mathbf{R}^{3}$ and is such that the limits as $z \rightarrow p$ and as $z \rightarrow q$ of $I_{3}(z)$ exist uniformly as extended real numbers.

Received by the editors August 28, 1990.

1991 Mathematics Subject Classification. Primary 53A10, 53C42; Secondary 49F10, 53A05.

Key words and phrases. Minimal surfaces, Nitsche's conjecture, catenoid, bounded Gaussian curvature, finite total curvature.

This paper is part of a dissertation submitted in partial fulfillment of the requirements for the degree of Doctor of Philosophy at the University of Notre Dame, South Bend, Indiana, 1990. It was presented at the 93rd Summer Meeting of the American Mathematical Society on August 10, 1990. 
Then $M$ is of finite total curvature. In particular, if $M$ is embedded, then $M$ is a catenoid.

\section{INTRODUCTION}

We begin with the Weierstrass representation of minimal surfaces [O]

Theorem. Let $\Delta \subseteq \mathbf{C}$ be an open connected subset. Let $f: \Delta \rightarrow \mathbf{C}$ be holomorphic and $g: \Delta \rightarrow \mathrm{C} \cup\{\infty\}$ be meromorphic. Suppose that at each pole of $g$ of order $m$ there is a zero of $f$ of order $2 m$ and that $f\left(1-g^{2}\right)$, if $\left(1+g^{2}\right)$, and $f g$ have no real periods. Then there is a minimal immersion $I=\left(I_{1}, I_{2}, I_{3}\right): \Delta \rightarrow \mathbf{R}^{3}$ given by

$$
I_{1}=\operatorname{Re} \frac{1}{2} \int_{*}^{z} f\left(1-g^{2}\right), \quad I_{2}=\operatorname{Re} \frac{i}{2} \int_{*}^{z} f\left(1+g^{2}\right), \quad I_{3}=\operatorname{Re} \int_{*}^{z} f g
$$

Conversely, every minimal surface is of this form.

The metric on this surface is given by $d s^{2}=\frac{1}{2}|f|^{2}\left(1+|g|^{2}\right)^{2}|d z|^{2}$. The function $g$ given in the representation is (up to stereographic projection) the Gauss map of the surface. A minimal surface is said to be complete if every curve converging to the boundary of $\Delta$ is of infinite length. The Gaussian curvature of a minimal surface given by a Weierstrass representation is

$$
K=-\left(\frac{4\left|g^{\prime}\right|}{|f|\left(1+|g|^{2}\right)^{2}}\right)^{2}
$$

We will denote $\mathbf{C}-\{0\}$ by $\mathbf{C}^{*}$. It will be useful in our proofs to know what happens when we take $f g=1 / z$ in the Weierstrass representation

Proposition 1. Suppose that in the Weierstrass representation we have a domain $\Delta=\mathbf{C}^{*}, f: \Delta \rightarrow \mathbf{C}$ holomorphic, and $g: \Delta \rightarrow \mathbf{C} \cup\{\infty\}$ meromorphic. If we require that $f g(z)=1 / z$ then neither $f$ nor $g$ have any poles or zeros in $\mathbf{C}^{*}$. Proof. Immediate.

Under certain circumstances (stated in Lemma 3), we can restrict our attention to minimal surfaces generated by a small class of $\{f, g\}$ 's and to the single domain $\mathbf{C}^{*}$. We begin with two preliminary lemmas.

Lemma 1. Let $\Delta$ be a Riemann surface that is homeomorphic to $S^{2}-\{p, q\}$ with $U: \Delta \rightarrow \mathbf{R}$ harmonic. Suppose $\lim _{z \rightarrow p} U(z)=-\infty$ and $\lim _{z \rightarrow q} U(z)=\infty$. Then $\Delta$ is conformally equivalent to $\mathbf{C}^{*}$.

Proof. Observe that $\pi_{1}(\Delta)=\mathrm{Z}$. Let $\widetilde{\Delta}$ be the (holomorphic) universal cover of $\Delta$. Then $\tilde{\Delta}$ is conformally equivalent to either $\mathbf{C}$ or $D=\{|z|<1\}$. In the first case, since $\pi_{1}(\Delta)=\mathbf{Z}$, it follows that $\Delta$ is biholomorphic to $C$ modulo a translation group; that is, $\Delta$ is conformally $\mathrm{C}^{*}$.

We proceed to show that $\widetilde{\Delta}$ could not be $D$. Again, since $\pi_{1}(\Delta)=\mathbf{Z}$, $\Delta$ is conformally $D$ modulo $[g]$, where $g$ is a complex automorphism of $D$ without fixed points. It follows that, in the classical terminology, $g$ is hyperbolic if $g$ has two fixed points on $\partial D$ or parabolic if it has one fixed point. In the hyperbolic case, the fundamental region for the group $[g]$ is the complement 
of two disks in $D$ tangential to the fixed points on $\partial D$. In the parabolic case, the fundamental region lies between two geodesics emanating from the fixed point. Label the fundamental region (in both cases) $R$. Let $\rho: D \rightarrow \Delta$ be the natural projection, and note that $\tilde{U}=U \circ \rho: R \rightarrow \mathbf{R}$ is harmonic and tends to $\pm \infty$ on an arc of $\partial D$. Let $\widetilde{V}$ denote the harmonic conjugate of $\widetilde{U}$ on $D$. Now either $f_{1}=e^{(\widetilde{U}+i \widetilde{V})}$ or $f_{2}=e^{-(\widetilde{U}+i \widetilde{V})}$ tends to zero on this arc. By the reflection principle this holomorphic function is identically zero on $D$. This is a contradiction. Q.E.D.

Lemma 2. Let $U: \mathbf{C}^{*} \rightarrow \mathbf{R}$ be harmonic, and suppose that $\lim _{|z| \rightarrow 0} U(z)=-\infty$ and $\lim _{|z| \rightarrow \infty} U(z)=\infty$. Then $U\left(r e^{i \theta}\right)=a \log (r)+b$ where $a, b \in \mathbf{R}$.

Proof. By elementary complex analysis.

Lemma 3. Let $M$ be a complete minimal surface satisfying condition (ii) of Theorem 1 and such that $I_{3}: S^{2}-\{p, q\} \rightarrow \mathbf{R}$ is surjective. Then, up to translation, homothety, and relabeling of $p$ and $q$, we have that $M$ is given by a Weierstrass representation where: (1) $\Delta=\mathbf{C}^{*}$ and (2) $f g(z)=1 / z$.

Proof. Clearly we have $\lim _{z \rightarrow p} I_{3}(z)=-\infty$ and $\lim _{z \rightarrow q} I_{3}(z)=\infty$, up to relabeling of $p$ and $q$. By Lemma $1, S^{2}-\{p, q\}$ is conformally equivalent to $\mathbf{C}^{*}$. Thus in the Weierstrass representation we may take $\Delta=\mathbf{C}^{*}$. So we might as well have started with $M$ being a minimal immersion of $\mathbf{C}^{*}$ with $I_{3}: \mathbf{C}^{*} \rightarrow \mathbf{R}$ satisfying $\lim _{|z| \rightarrow 0} I_{3}(z)=-\infty$ and $\lim _{|z| \rightarrow \infty} I_{3}(z)=\infty$. By Lemma $2, I_{3}\left(r e^{i \theta}\right)=a \log (r)+b$ for some $a, b \in \mathbf{R}$.

Thus $M$ can be given in the Weierstrass representation by $\widetilde{I}=\left(\widetilde{I}_{1}, \widetilde{I}_{2}, \widetilde{I}_{3}\right)$ : $\mathbf{C}^{*} \rightarrow \mathbf{R}^{3}$, where we have taken $\tilde{I}_{3}(z)=I_{3}(z)$. That is, $I_{3}(z)=a \log (r)+b=$ $\widetilde{I}_{3}(z)=\operatorname{Re} \int_{*}^{z} f g d z$. After translation, we may discard $b$. We are left with $\widetilde{I}_{3}(z)=a \log (r)$. Applying a homothety (by $1 / a$ ) yields

$$
\frac{1}{a} \widetilde{I}_{3}=\frac{1}{a} \operatorname{Re} \int_{*}^{z} f g d z=\log (r) .
$$

We can assume $a=1$, and therefore take $f g=1 / z$. Q.E.D.

We will also use a result of Lehto and Virtanen on the growth aspects of meromorphic functions. Let $h: \Delta \subseteq \mathbf{C} \rightarrow \mathbf{C} \cup\{\infty\}$ be a meromorphic function, where $\Delta$ is open and connected. The spherical derivative of $h$ is defined as

$$
\rho(h(w))=\frac{\left|h^{\prime}(w)\right|}{\left[1+|h(w)|^{2}\right]} .
$$

Lemma 4. Let $h$ be a meromorphic function in a neighborhood of an essential singularity at infinity that satisfies the inequality: $\lim _{\sup _{z \rightarrow \infty}}|z| \rho(h(z))<\infty$. Then $h$ cannot omit any value.

Proof $[\mathrm{LV} 2$, p. 7,8]. Let $\gamma$ be a Jordan path in $U$ tending to $\infty$. Then $\alpha$ is said to be an asymptotic value of $h$ at $\infty$ if $h(z) \rightarrow \alpha$ as $z \rightarrow \infty$ along $\gamma$. Suppose $h$ omits the value $\alpha$. Then by Iversen's Theorem [No, p. 4], $\alpha$ is an asymptotic value at infinity along a Jordan path $\gamma$. By the above theorem, $h$ is normal in $U$ slit along the path $\gamma$. By Theorem 2 of [LV1, p. 53] and the 
remark that follows, $h$ converges uniformly in $U-\{\gamma\}$ toward $\alpha$, no matter how $z$ goes to $\infty$. This contradicts the hypothesis that $h$ has an essential singularity at $z=\infty$. Hence $h$ cannot omit any value. Q.E.D.

If $h$ omits the value 0 and satisfies the inequality, then it could not have had an essential singularity at $z=\infty$.

\section{The RESULT}

The following theorems are needed for the proof of Theorem 1.

Theorem (Jorge-Meeks [JM]). The catenoid is the only embedded complete minimal annulus in $\mathbf{R}^{3}$ that has finite total curvature.

Theorem (Xavier [X]). Let $M^{2} \subset \mathbf{R}^{3}$ be a complete minimal surface that is nonflat and of bounded Gaussian curvature $(|K|<C)$. Then the convex hull of $M$ is $\mathbf{R}^{3}$.

Corollary. Let $M$ be a complete minimal immersion satisfying conditions (i) and (ii) of Theorem 1. Then $I_{3}$ is surjective.

Proof (of Theorem 1). By the corollary to Xavier's Theorem, $I_{3}$ is surjective. By Lemma 3, we have that, up to translation, homothety, and the relabeling of $p$ and $q, M$ is given in the Weierstrass representation by $\Delta=\mathrm{C}^{*}$ and $f g(z)=1 / z$. By Proposition $1, f$ has no zeros in $\mathbf{C}^{*}$. Thus we can set $\left(\frac{1}{|f|}\right)=|z g|$ and $z=r e^{i \theta}$. Setting $h(z)=g(z)^{2}$, the above expression for Gaussian curvature becomes

$$
K=-\left(\frac{2 r\left|h^{\prime}\right|}{(1+|h|)^{2}}\right)^{2}
$$

Thus,

$$
\begin{aligned}
|K|<C \Rightarrow\left(\frac{r\left|h^{\prime}\right|}{(1+|h|)^{2}}\right)<C & \Longleftrightarrow\left(\frac{\left|h^{\prime}\right|}{(1+|h|)^{2}}\right)<\frac{C}{r} \\
& \Longleftrightarrow\left(\frac{\left|h^{\prime}\right|}{1+|h|^{2}}\right)<\frac{C_{1}}{r} .
\end{aligned}
$$

In terms of the spherical derivative, we have

$$
|K|<C \Rightarrow|z| \rho(h(z))<C_{1} \quad \forall z \in \mathbf{C}^{*} .
$$

By Proposition 1, $g$ (and hence $h$ ) has no zeros or poles in $\mathbf{C}^{*}$. All that remains is to check for singularities at 0 and $\infty$. Suppose that $h$ has an essential singularity at $\infty$. We have just shown that

$$
|z| \rho(h(z))<C \quad \forall z \epsilon \mathbf{C}^{*}, \quad \text { so } \limsup _{|z| \rightarrow \infty}|z| \rho(h(z))<C<\infty .
$$

Thus by Lemma $4, h$ cannot omit any value. This is a contradiction since we have shown that $h$ omits zero. Hence $h$ could not have had an essential singularity at infinity. Suppose now that $h$ has an essential singularity at $z=0$. 
Then by the inversion $z \mapsto \frac{1}{z}$ we have that $h\left(\frac{1}{z}\right)$ has an essential singularity at $\infty$. Observe that

$$
\left(\frac{|z|\left|h^{\prime}(z)\right|}{1+|h(z)|^{2}}\right)<C \quad \forall z \in \mathbf{C}^{*} \Longleftrightarrow\left(\frac{|z|\left|\left(h\left(\frac{1}{z}\right)\right)^{\prime}\right|}{1+\left|h\left(\frac{1}{z}\right)\right|^{2}}\right)<C \quad \forall z \epsilon \mathbf{C}^{*}
$$

By the above argument, $h$ could not have had an essential singularity at zero.

We have that $h$ has a pole or removable singularity at $z=0$ and $z=\infty$. Hence $h$ is meromorphic on $\mathbf{C} \cup\{\infty\}$ and therefore rational [F, p. 11]. This implies that $g$ is also rational. The image of $\mathbf{C}^{*}$ under $g$ has finite area on $S^{2}$; that is, we have a complete minimal surface $M$ of finite total curvature.

If $M$ is embedded, apply the result of Jorge-Meeks [JM] to conclude that $M$ is a catenoid. Q.E.D.

We have, as a corollary to Theorem 1, that Nitsche's conjecture holds under the hypothesis of bounded Gaussian curvature.

Theorem 2. Let $M$ be a complete minimal immersion satisfying conditions (i) and (ii) of Theorem 1. Suppose that the level sets of $I_{3}: S^{2}-\{p, q\} \rightarrow \mathbf{R}$ are homeomorphic to $S^{1}$. Then $M$ is a catenoid.

Proof. $M$ is embedded, so by Theorem 1, $M$ is a catenoid. Q.E.D.

Rosenberg has given a result on the unwillingness of the catenoid to be perturbed [R]. Certain definitions are needed and are given as follows. Let $T_{\varepsilon}$ be a tubular neighborhood of radius $\varepsilon>0$ about $M \subset \mathbf{R}^{3} . \widetilde{M}$ is said to be an $\varepsilon-C^{1}$ variation of $M$ if $\widetilde{M}$ is a graph over $M$ which is in $T_{\varepsilon}$ and is $C^{1}$ close to $M$. This means $\widetilde{M}$ is pointwise $\varepsilon$-close to $M$ in each fibre of $T_{\varepsilon}$ and in the tangent planes as well. $M$ is said to be isolated if for some $\varepsilon>0$, the only $\varepsilon-C^{1}$ variations of $M$ differ from $M$ by an isometry of $\mathbf{R}^{3}$. With these definitions, he showed that the catenoid is $\varepsilon-C^{1}$ isolated.

Theorem 1 is of the same flavor as this result and leads toward the conjecture that the catenoid is not only $\varepsilon-C^{1}$ isolated but is in fact $\varepsilon-C^{0}$ isolated.

\section{REFERENCES}

[CHM] M. Callahan, D. Hoffman and W. H. Meeks, III, Embedded minimal surfaces with an infinite number of ends, preprint.

[F] O. Forster, Lectures on Riemann surfaces, Graduate Texts in Math., vol. 81, Springer-Verlag, New York, 1981.

[JM] L. P. M. Jorge and W. H. Meeks, III, The topology of complete minimal surfaces of finite total Gaussian curvature, Topology 22 (1983), 203-221.

[LV1] O. Lehto and K. I. Virtanen, Boundary behaviour and normal meromorphic functions, Acta Math. 97 (1957), 47-65.

[LV2] $\longrightarrow$ On the behavior of meromorphic functions in the neighbourhood of an isolated singularity, Ann. Acad. Sci. Fenn. Ser. A I Math. 240 (1957), 3-9.

[Ni] J. C. C. Nitsche, A characterization of the catenoid, J. Math. Mech. 11 (1962), 293-302.

[No] K. Noshiro, Cluster sets, Springer-Verlag, Berlin, 1960.

[O] R. Osserman, A survey of minimal surfaces, 2nd ed., Dover Publications, New York, 1986.

[R] H. Rosenberg, Deformations of complete minimal surfaces, Trans. Amer. Math. Soc. 295 (1986), 475-489. 
[S] R. Schoen, Uniqueness, symmetry, and embeddedness of minimal surfaces, J. Differential Geom. 18 (1983), 791-809.

[X] F. Xavier, Convex hulls of complete minimal surfaces, Math. Ann. 269 (1984), 179-182.

Department of Mathematics and Computer Science, John Carroll University, UniVERSITY HEIGHTS, OHIO 44118 